\title{
Spatio-temporal and stochastic modelling of severe acute respiratory syndrome
}

\author{
Poh-Chin Lai ${ }^{1}$, Kim-Hung Kwong ${ }^{2}$, Ho-Ting Wong ${ }^{2}$ \\ ${ }^{1}$ Department of Geography, The University of Hong Kong, Hong Kong, People's Republic of China; ${ }^{2}$ Formerly \\ at the Department of Geography, The University of Hong Kong, Hong Kong, People's Republic of China
}

\begin{abstract}
This study describes the development of a spatio-temporal disease model based on the episodes of severe acute respiratory syndrome (SARS) that took place in Hong Kong in 2003. In contrast to conventional, deterministic modelling approaches, the model described here is predominantly spatial. It incorporates stochastic processing of environmental and social variables that interact in space and time to affect the patterns of disease transmission in a community. The model was validated through a comparative assessment between actual and modelled distribution of diseased locations. Our study shows that the inclusion of location-specific characteristics satisfactorily replicates the spatial dynamics of an infectious disease. The Pearson's correlation coefficients for five trials based on 3-day aggregation of disease counts for 1-3, 4-6 and 7-9 day forecasts were 0.570.95, 0.54-0.86 and 0.57-0.82, respectively, while the correlation based on 5-day aggregation for the 1-5 day forecast was 0.550.94 and $0.58-0.81$ for the 6-10 day forecast. The significant and strong relationship between actual results and forecast is encouraging for the potential development of an early warning system for detecting this type of disease outbreaks.
\end{abstract}

Keywords: infectious disease epidemiology, spatial modelling, estimating disease spread, SARS, geographical information system, early warning system, Hong Kong.

\section{Introduction}

The rapid transmission pace of infectious diseases today has attracted much attention from public health practitioners and institutes worldwide (Armelagos, 1998; Barrett et al., 1998; Satcher, 1995; WHO, 2003). The first transmissible disease of potential worldwide threat in the $21^{\text {st }}$ century was severe acute respiratory syndrome (SARS) that originated in Asia. From March to July 2003, the virus reached approximately 30 countries and over 8,400 people across the world were infected (WHO, 2003; Ali and Keil, 2006; Nelson, 2007). More recently, the 2009 epidemic of H1N1 influenza A, commonly known as "swine flu", penetrated international boundaries in record time (Barrett et al., 1998; WHO, 2003; Nelson, 2007). This disease began in Mexico with an outbreak in midMarch 2009 and had already spread to a dozen countries in the Americas, Europe, Middle East by April, even reaching as far as New Zealand. In early May 2009, the disease also appeared in the People's Republic of China (P.R. China) including Hong Kong, and South Korea.

Corresponding author:

Poh-Chin Lai

Department of Geography, The University of Hong Kong

Pokfulam Road, Hong Kong SAR, People's Republic of China

Tel. +852 3917-2830; Fax +852 2559-8994

E-mail: pclai@hku.hk
It is obvious that a more connected world and increased globalisation have expedited the disease transmission process. Hence, early identification of high-risk locations and a better spatial understanding of disease transmission dynamics (i.e. how a disease spreads within a population) are critical for targeted intervention strategies. With effective control measures such as early detection and targeted segregation, many infectious diseases can be contained (WHO, 2003; Connolly et al., 2004; Lau et al., 2004ab). Jefferson et al. (2008) felt that systematic education on personal hygiene and physical interruptions (e.g. immediate isolation of infected patients) are important in preventing rapid dispersal of respiratory diseases. Hence, an early identification of high-risk locations and a better spatial understanding of the disease transmission dynamics are critical for targeted intervention strategies at specific locations.

There has been progress in infectious disease modelling over the past three decades but the use of such models in public health decision-making has not been extensive. Broadly speaking, mathematical modelling is classified as either deterministic (models with no random variables) or stochastic (those with one or more random variables). Conventional, deterministic, epidemic models, such as the susceptible-infectedremoved (SIR) and susceptible-exposed/infectedremoved (SEIR) approaches (Anderson and May, 1991), reduce the population diversity to a few compartments relevant to the infection under examination. 
Such models often have fixed regulations to change (Aron, 2007) and focus on temporal or seasonal changes. They have the advantage of being simple with less stringent data requirement (Trottier and Philippe, 2000). Coen (2007), however, criticised deterministic models as "too simplistic to be realistic" mentioning that populations are usually regarded as homogeneous, which they seldom are. Besides, the models are often entirely temporal in nature and rely too much on the basic reproduction number $\left(\mathrm{R}_{0}\right)$. Various researchers (Dye and Gay, 2003; Ali et al., 2009) feel that deterministic models tend to neglect the variation in contact rates and/or heterogeneity in disease transmission. Contrary to the fact that certain communities may be less susceptible to infection, the typical, deterministic model suggests that an epidemic would ultimately infect the entire population regardless of where people live and who they are (Lipsitch et al., 2003; Shannon and Willoughby, 2004).

Other than the deterministic approach, some stochastic mathematical models have been adopted for early infectious outbreak detection. Held et al. (2005) proposed a forecast model based on Poisson branching using immigration and temporally dynamic variables for food- or water-borne infectious disease surveillance. This stochastic forecast model was tested by Sifat and Rayhan (2012) for the detection of measles epidemics through airborne transmission in Bangladesh. They found that the negative binomial model with both seasonality and auto-regressive components outperformed other Poisson models as a tool for forecast in terms of root mean square error. The characteristics of measles and its occurrence could be captured by the model through a "one-step-ahead" predication based on infection history in the past 12 months. This finding is consistent with those of Held et al. (2005) suggesting that the negative binomial model could be applied to other similar settings. Sharmin and Rayhan (2011) have also proposed a two-stage monitoring system for measles, where the system consisted of a stochastic seasonal autoregressive integrated moving average model followed by several statistical process-control charts using data on measles epidemics from September 2007 to August 2009. Forecasts one month in advance for the period closely mirrored the observed monthly values of the first two years. Overall, the system detected no measles outbreak during the period which matched the situation of no real epidemic given the high measles vaccination coverage at the time. Besides, spatial or gravity interaction and diffusion models have also been widely used in analysing airborne disease transmission (Camitz and Liljeros, 2006; Viboud et al., 2006a, 2006b; Berry et al., 2008). These studies illustrate that the spread of an airborne disease is a complex function of host movement governed by social and demographic structures and susceptibility. Indeed, spatial heterogeneity involves more than simple distance or adjacency relationships but also engages local mechanisms of spatial mixing manifested by accessibility and potential destination (Lai et al., 2004). In terms of infectious disease modelling, there is event-driven versus individual-based stochasticity. Knowledge and awareness on disease dynamics, environmental hygiene, crowding, transport connectivity, medical facilities and human behaviour can surely influence the transmission patterns of an airborne infectious disease (Connolly et al., 2004; Lau et al., 2004ab; Bowen and Laroe, 2005; Meng et al., 2005; Fang et al., 2009).

We describe here a model, exemplified by the SARS episodes of Hong Kong in 2003, which includes spatial and stochastic factors relevant to infectious disease epidemiology and document the modelling approaches that incorporate these factors mathematically.

\section{Materials and methods}

\section{Study site}

The study took place in Hong Kong with the exclusion of non-populated areas such as country parks, cargo terminals, fire ranges, water bodies and uninhabited islands. A spatial grid of consisting of $500 \mathrm{x}$ 500 metric cells was established with the study area covering 2,221 cells $(12 \%$ of the total area of Hong Kong) out of all the 18,510 cells. It covered all built and populated areas in Hong Kong (Fig. 1). This particular resolution was selected by considering various factors: (i) subject privacy; (ii) forecast accuracy; and (iii) computer processing capacity. Indeed, testing the $150 \mathrm{~m} \times 150 \mathrm{~m}$ resolution indicated that the computing power was insufficient to complete the processing required. Moreover, finer resolutions are overly sensitive resulting in instability of the forecast accuracy.

\section{Data}

We employed disease data of the 2003 SARS outbreak in Hong Kong that occurred between 18 February 2003 and 2 June 2003 inclusive. The disease locations $(n=1,075)$ were geocoded at the residential addresses of infected individuals and the data transferred into the grid format. 


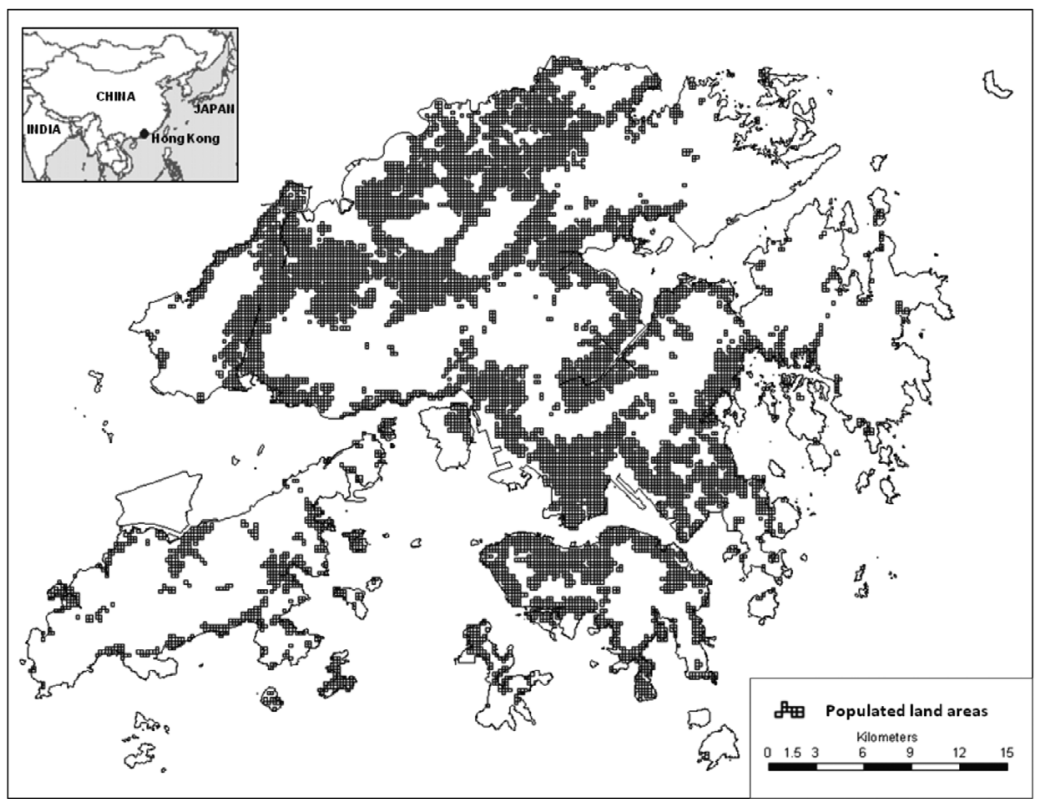

Fig. 1. Study area in Hong Kong (500 x 500 metric grid).

Population census data were obtained from the Hong Kong Census and Statistics Department (2001). It is noteworthy that the spatial variation of Hong Kong's population is very large, ranging between 1 and 42,808 between the populated cells in the study. Incorporating the population factor into the model is essential because it has been shown that areas of high population density facilitate disease spread and increase the likelihood of local outbreaks (Kwong and Lai, 2010). Other location-specific characteristics were computed based on data from various sources and primarily from the geocommunity database (containing locations of social amenities) available from the Hong Kong Lands Department.

\section{Model development}

The transmission dynamics of SARS have been examined using the SIR/SEIR models (Donnelly et al., 2003; Dye and Gay, 2003; Ali et al., 2009). Although the SEIR model deals primarily with temporal transmission without considering spatial interaction, it does have two useful features. Firstly, it is based on assumptions that are approximately true for many diseases (Anderson and May, 1991). Secondly, it has only four parameters, a feature which keeps the model relatively simple and introduces less uncertainty.

To model spatial variation of infectivity in a geographical information system (GIS), the spatial dimension can be introduced by assuming SEIR with a specific, initial, reproductive number $\left(\mathrm{R}_{0}\right)$ to occur in a closed population arranged in a $500 \times 500$ metric grid
(Fig. 1). We employed an in-house programme to generate results, which could be imported into ArcGIS (ESRI; Redlands, USA) for subsequent processing. The grid approach considers each cell a unique entity, which makes the system capable of modelling spatial heterogeneity within a closed population. Disease transmission dynamics were modelled in a GIS by considering not only "within-cell” but also "cross-cell" transmission (Fig. 2). The latter was anchored on an infected/pivot cell and adjusted by two factors with spatial randomness: (i) spatial proximity (i.e. the distance between residences) and (ii) social mixing (i.e. the availability of social amenities which play a significant role in manoeuvring the flow of people). This process is detailed in subsequent paragraphs and shown in Figs. 3-5.

We made use of the epidemiological parameters of SARS estimated by Riley et al. (2003), i.e. infectious period $(v)$, incubation period $(\sigma)$ and effective contact rate $(\beta)$ derived from the basic reproduction number $\left(\mathrm{R}_{0}\right)$ for the closed population of Hong Kong. On the first day of a disease outbreak (i.e. $t=0$, the numbers of susceptible (S), exposed (E), infected (I) and removed $(\mathrm{R})$ individuals were determined for each cell by the temporal SEIR model based on the above epidemiological parameters. Spatial heterogeneity is thus represented by the grid structure where each cell varies in terms of S, E, I and R depending on status and residential address. For each cell, the exposed population at time $t$ based on the SEIR approach shown in equation 1 is computed as a function of susceptible and infected individuals within the total population $(\mathrm{N})$ 


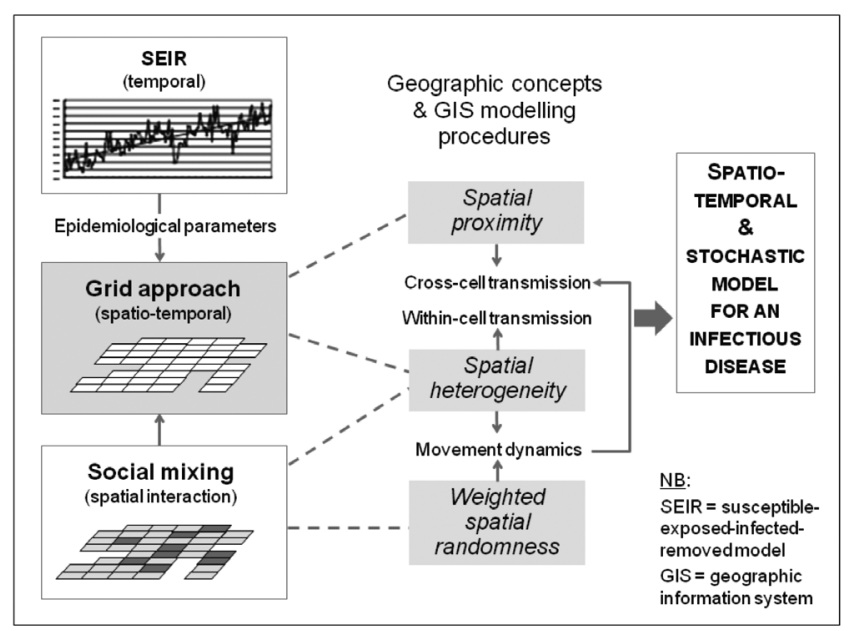

Fig. 2. Conceptual design and model development.

and considering the effective contact rate but excluding the part of the population that became infectious, i.e. the exposed population beyond the incubation period. The differential equations for other compartments of susceptible, infected, and removed parts of the population are derived accordingly and the sum total of all S-E-I-R components is held constant as the total population of each cell.

$$
\begin{gathered}
\frac{d E}{d t}=\beta \frac{S I}{N}-\sigma E \\
\frac{d S}{d t}=-\beta \frac{S I}{N} \\
\frac{d I}{d t}=+\sigma E-v I \\
\frac{d R}{d t}=+v I \\
S+E+I+R=N
\end{gathered}
$$

Disease numbers for each cell in subsequent days are estimated by considering two factors of spatial heterogeneity for "within-cell" transmission and spatial proximity for "cross-cell" transmission (Fig. 3). Spatial heterogeneity regards localised SEIR component values to have an impact on the disease contact rate. For example, larger susceptible, infected, and exposed population compartments would be expected to increase the chance of "within-cell" disease transmission as well as disease transmission to neighbour cells but at varying degrees. Spatial proximity considers three kinds of cell in the modelling of "cross-cell" transmission (Figs. 3 and 4):

(i) pivot cell - a moving cell that visits every cell in the grid;

(ii) neighbours - cells surrounding the pivot cell ( 3 to 8 first-order neighbours); and

(iii) other cells - cells selected by random processes.

Besides, the population in the pivot cell is more like(1) ly to be infected by infected individuals living in neigh-

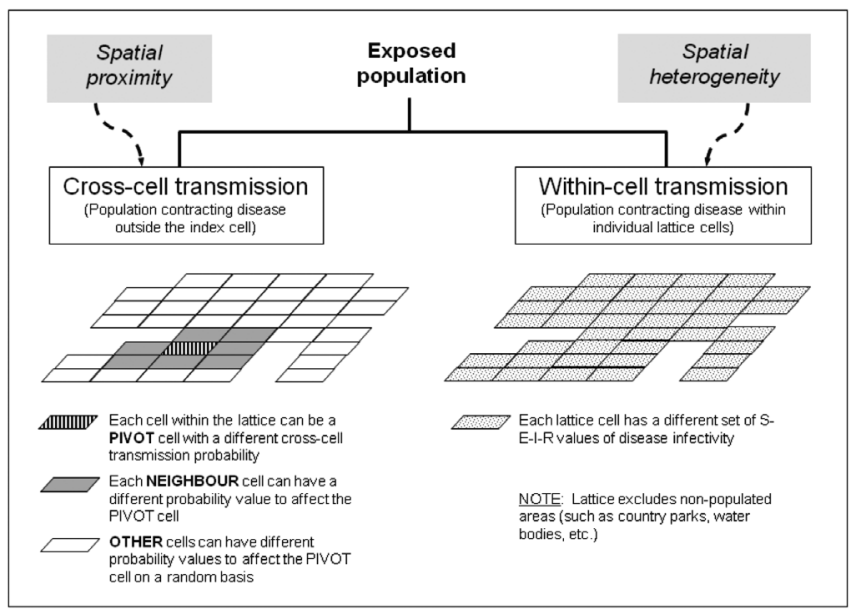

Fig. 3. Components of exposed population. 


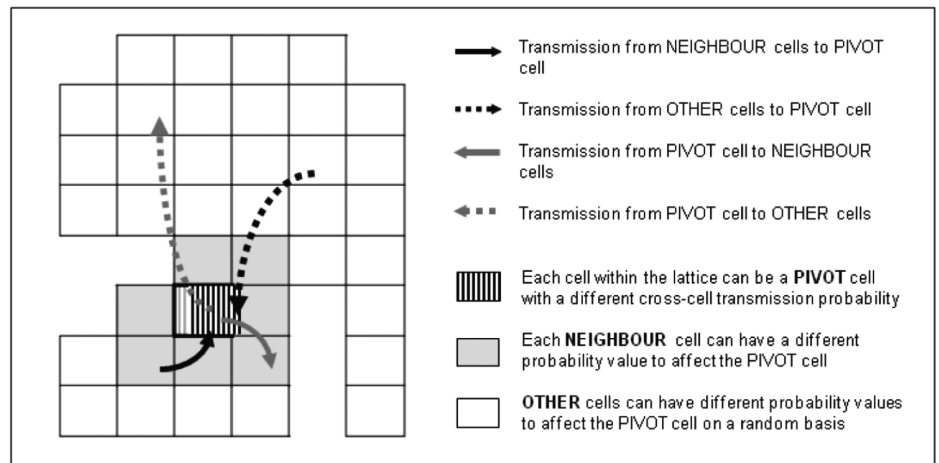

Fig. 4. Mechanisms of "cross-cell" disease transmission.

bour cells of close proximity ("within-cell" transmission). Infection beyond the living environment (e.g. from co-workers or school mates or people on the streets) is also likely and this event is modelled through a weighted random process.

"Cross-cell" transmission for each pivot cell has two constituents: exported and imported transmission as described by equation 2 . For exported transmission, our model assumes individuals in the pivot cell to exert two levels of interaction: a higher level with residents in neighbour cells and a lower level with other cells (Fig. 4). Following the geographical law introduced by Tobler (1970) that "everything is related to everything else, but near things are more related than distant things", neighbour cells closer to a pivot, or an infected cell, were assigned a higher probability than random cells further away. The same argument is made for imported transmission, in which a higher probability of interaction with a pivot cell comes from a neighbour as opposed to random cells.

$$
\frac{d E}{d t}=\beta \frac{S I}{N}-\underbrace{E_{o}}_{\begin{array}{c}
\text { Exported, cross-cell } \\
\text { transmission }
\end{array}}+\underbrace{E_{i}}_{\begin{array}{c}
\text { Imported, cross-cell } \\
\text { transmission }
\end{array}}-\sigma E
$$

Spatial stochasticity is emulated by assigning to each cell a probability value of social interaction given location-specific characteristics (Fig. 5). This type of characteristics can be useful as indicators, e.g. for population density or destinations that attract crowds (including retail/shopping centres, business establishments, entertainment places, hospitals/clinics and transit centres such as bus and rail stations). Locationspecific characteristics may vary from place to place (Smith et al., 2005; Eisenberg et al., 2007) but their existence in Hong Kong has been shown to correlate in some ways with disease incidence (Kwong and Lai, 2010). Combined, these factors reflect the degree of social mixing. The aggregated probability values can be sorted in ascending (or descending) order to construct linear and progressive arrangements or a weight list upon which random selection is applied.

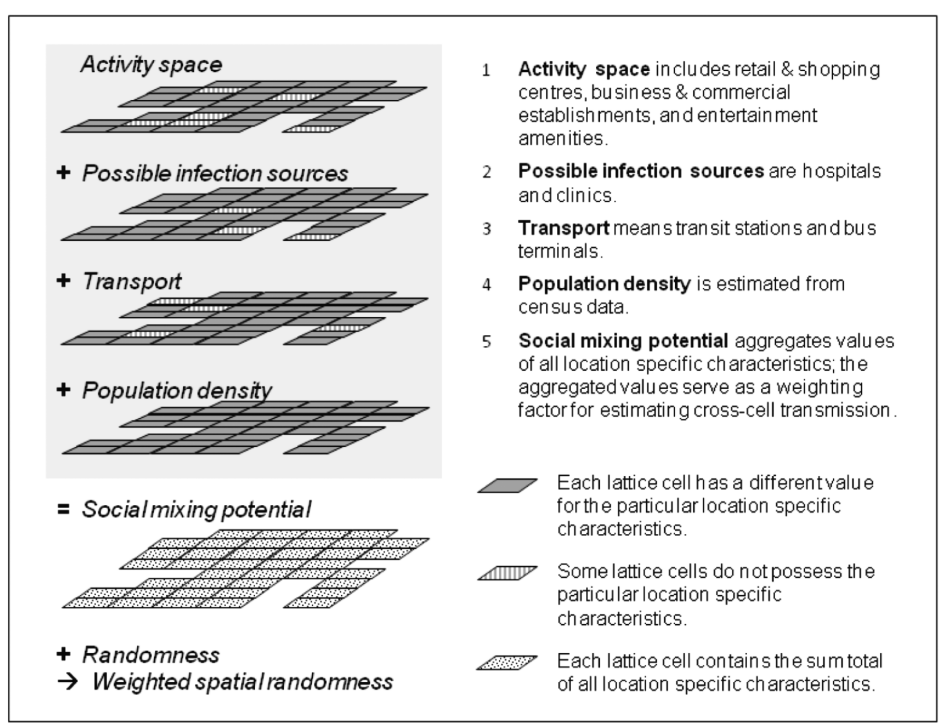

Fig. 5. Social mixing potential and weighted spatial randomness. 
The stochastic mechanism in our model was introduced for the estimation of "cross-cell" transmission (both exported and imported) with neighbour and other cells. Here, our model allows proportions of the exposed population within a pivot cell to contract disease from its neighbour and other cells randomly chosen by the system but in consideration of the social mixing potential. The probability of "within-cell" transmission, "cross-cell" transmission to neighbour cells, and "cross-cell" transmission to random cells is adjusted according to advice from medical professionals and their expert knowledge about the disease. As the total number of infected cases is controlled by the value of $\mathrm{R}_{0}$, the proportion of one type of transmission will affect the other two. Incidentally, "within-cell" transmission is obtained by subtracting "cross-cell" transmission from the total transmission. Our spatiotemporal and stochastic model operates with four fundamental assumptions as follows:

(i) each disease case is equal;

(ii) each disease case yields a similar number of secondary cases;

(iii) the disease incubation period remains constant over the entire simulation/epidemic period; and

(iv) quarantined or removed patients are no longer infective.

\section{Model validation}

The total number of people on the day symptoms first appeared was considered as the actual number of SARS-infected cases for that date. The study employed the onset date of symptoms because a SARS patient is considered infectious from this day until 7-14 days later (Donnelly et al., 2003). Using the onset date thus ensures inclosure of the beginning of the infectious period, even if the diagnosis is not confirmed until later. This actual count was compared with the number of newly infected cases estimated by our spatio-temporal SEIR model. Five runs of the model were performed to simulate the disease spread for a period of 20 days following day 0 . Day 1 of the simulation would use disease cases reported in the preceding 10 days and the predicted disease cases would be the sum of estimated new cases and reported cases for the preceding 10 days. A 10 -day moving window was applied for days 2-20. We applied the model using 3, 8, 13, 18 and 23 March 2003 as day 0 . The model validity was assessed visually by plotting the cumulative results of actual and modelled counts on a map. Furthermore, Pearson's correlation coefficient $(r)$ was adopted to evaluate the modelled results for both temporal and spatial stabilities
(Kwong and Lai, 2010).

Pearson's correlation was calculated by treating each grid cell as a record $(2,221$ cells in total) to derive a single correlation for a time range. For example, the spatial accuracy for day 1-3 forecasts incurs daily cases in each cell for three consecutive days. Three grid meshes, one for each of of the three days, were added together to yield a single one of 3-day forecast aggregation. A single correlation can thus be calculated to assess the spatial accuracy of the forecast for the 1-3 day period by comparing the cumulative forecast and actual cases in each cell for the selected time range of 3 days. Likewise, the temporal stability of the forecast model can be assessed by computing correlation values for different time ranges. For example, correlation values for days 1-3, 4-6 and 7-9 can be derived to examine the temporal effect of forecast accuracy. If the series of forecasts consistently yields high correlation for all three time ranges (days 1-3, 4-6 and 7-9), then the results would signify temporal stability of the model, whereas irregular correlation would indicate the opposite.

\section{Results}

Fig. 6 shows the cumulative results of the 20-day simulation with 3 March 2003 as day 0. The disease diffused from several origins to other areas in Hong Kong but the spread was not very severe at this early stage. Results of this run even suggested a diminishing trend, i.e. that the epidemic would vanish in about half a month after day 0 if no additional adjustment were made on newly infected cases through disease surveillance.

The ability to detect disease outbreaks a few days in advance is sufficient to trigger outbreak management or formulation of infection control measures. Therefore, results of Pearson's correlation coefficient ( $\mathrm{r}$ ) based on 3- and 5-day aggregation of disease counts were used to facilitate analysis, as shown in Table 1 . The values indicated a statistically significant correlation (at least at $\mathrm{P}$ $<0.01$ ) between the actual and modelled counts for up to 10-day forecasts. Among the five trials described in section 2.2, the $\mathrm{r}$ values based on 3-day aggregation of disease counts for 1-3, 4-6 and 7-9 day forecasts were $0.57-0.95,0.54-0.86$ and $0.57-0.82$, respectively. Similarly, the r-values based on 5-day aggregation of disease counts for 1-5 and 6-10 day forecasts were 0.550.94 and $0.58-0.81$, respectively. The correlation values generally indicated decreasing performance with increasing number of days of forecast. This finding is not unreasonable because of increasing externalities and uncertainties the further we move into the future. 


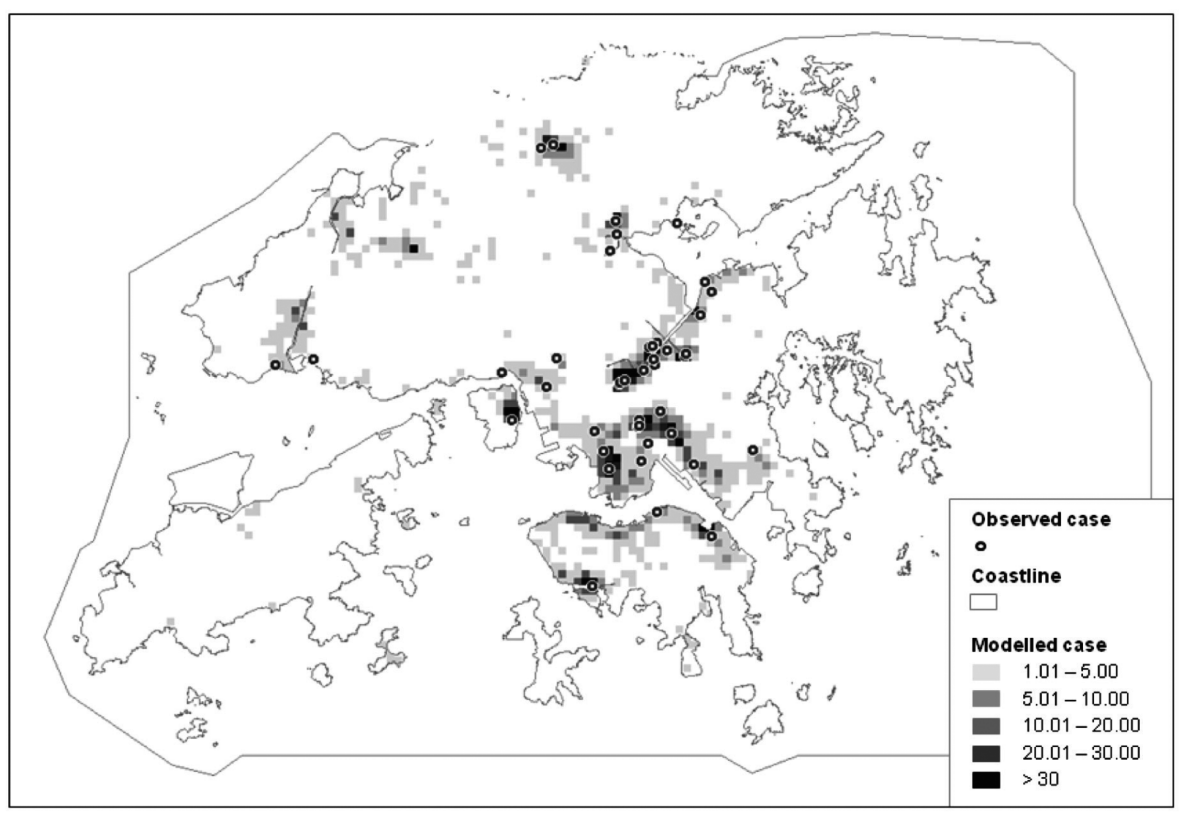

Fig. 6. Modelled and actual SARS cases showing cumulative results for the simulation period (3-23 March 2003).

\section{Discussion}

We have constructed a spatial-temporal stochastic model to simulate the spatial diffusion of an infectious disease in a compact, urban setting. The model has three unique features: (i) it makes use of the wellestablished SEIR epidemiological model to account for the temporal aspect of disease transmission; (ii) it partitions disease changes over time into discrete geographical units to account for the spatial aspect of disease transmission; and (iii) it incorporates locationspecific characteristics to account for the spatial interaction, which is often neglected in studies of disease transmission. In the modelling of a highly infectious disease like SARS, the model's predictability is greatly affected by the ability to provide "accurate" counts and the whereabouts of the infected cases to enable instant adjustments of input parameters. For example, timely adjustment of parameters for the "within-cell" and "cross-cell" transmission is essential because the proportion of "within-cell" and "cross-cell" transmission is reciprocal, as described in the model development section. Overestimating the "within-cell" transmission may result in false positive forecast of local disease outbreaks whereas overestimating the "crosscell" transmission may yield anomalous forecast of more than expected spatial spread of the disease without observable local outbreaks. This condition suggests the need to augment field epidemiologic data collection and improve health informatics by effective disease reporting that can offer rapid and close to realtime accounts of disease occurrence.

The use of available, retrospective data in the model development allowed us to verify prediction accuracy by comparing modelled results against actual counts. Our model consistently yields reliable results for predictions up to 10 days. This is encouraging as the goal of disease modelling, at least in the case of communicable diseases, is to offer early and reliable prediction of transmission sites and target locations to devise

Table 1. Performance results by different temporal aggregations $(\mathrm{n}=2,221)$.

\begin{tabular}{|c|c|c|c|c|c|c|}
\hline \multirow{2}{*}{$\begin{array}{l}\text { Days of } \\
\text { aggregation }\end{array}$} & \multirow{2}{*}{$\begin{array}{l}\text { Days of } \\
\text { forecast }\end{array}$} & \multicolumn{5}{|c|}{ Day $0(t=0)$} \\
\hline & & 3-Mar & 8-Mar & 13-Mar & 18-Mar & 23-Mar \\
\hline \multirow{3}{*}{3 days } & Day 1-3 & $0.64 * * *$ & $0.77 * * *$ & $0.79 * * *$ & $0.95 * * *$ & $0.57 * *$ \\
\hline & Day 3-6 & $0.58 * *$ & $0.79 * * *$ & $0.86 * * *$ & $0.86 * * *$ & $0.54 * *$ \\
\hline & Day 7-9 & $0.82 * * *$ & $0.73 * * *$ & $0.63 * * *$ & $0.57 * * *$ & $0.67 * *$ \\
\hline \multirow{2}{*}{5 days } & Day 1-5 & $0.67 * * *$ & $0.82 * * *$ & $0.89 * * *$ & $0.94 * * *$ & $0.55 \%$ \\
\hline & Day 6-10 & $0.81 * * *$ & $0.75 * *$ & $0.65 * *$ & $0.58 * *$ & $0.68 * * *$ \\
\hline
\end{tabular}

$* \mathrm{P}<0.05 ; * \mathrm{P}<0.01 ; * * \mathrm{P}<0.001$ 
public health countermeasures that can effectively contain the disease from spreading. An accurate, albeit somewhat short-term prediction can buy time for public health officials to consider possible control alternatives that prevents local outbreaks from growing into a full-blown epidemic. Our research thus offers essential foundation for the development of an early warning system for any infectious disease, e.g. it would be applicable for the web-based system that was developed for real-time monitoring of schistosomiasis transmission (Yang et al., 2012).

Considering the implications of operational efficiency with prediction accuracy, we can highlight several priorities for future research. Firstly, it has been shown that empirical, social contact data provide a better fit to disease cases (Meade 1997; Lau et al., 2004b; MacPherson et al., 2009). We can thus employ census data on cross-district commuting to work and school to improve mobility accounts that reflect the dynamics of spatial interaction. Secondly, we could relax assumptions 1 and 2 of the model that stipulate equal weight and effects of all disease cases. For example, the weight of a known index patient can be adjusted and contact tracing (by means of work address and occupation) incorporated in the model to detect subsequent spread. Thirdly, assumption 3 (stating that the disease incubation period will remain constant over the entire epidemic period) could be more flexible. This modification would permit the model to consider diseases with similar attributes but allowing for changes in epidemiological settings, such as the $\mathrm{R}_{0}$ value. Fourthly, there is a need to include options in the model to simulate the effects of intervention and control measures. This modification would enable decision makers to explore, with some degrees of certainty, the effectiveness of various countermeasures. Fifthly, to enable our model to be applied in a prospective, instead of retrospective setting, there is a need to develop a statistical tool to calculate confidence intervals of the transmission results without prior knowledge of the actual disease outcome. Finally, more sophisticated, geostatistical tests could be adopted to strengthen assessment of spatial forecast accuracy in addition to temporal forecast accuracy (Lai et al., 2009).

The research has prompted us to rethink two operating principles: (i) the sole use of residential addresses of infected individuals as disease locations in view of the mobile population, and (ii) the effective grid size to model activity space. Present epidemiological data collection does not have provision to collect more than one address (such as work locations for the employed; school addresses for school-aged children; and popular locations for social gathering). Given that the primary transmission route of influenza viruses is spread by airborne droplets during close contact, vigorous contact tracing of contagious patients during the initial phase of an epidemic is necessary. These data are critical to refining the patterns of social interaction thereby enhancing predictability of the disease model. We are hopeful that the advent of mobile computing and anonymous location tracking technology can promise more effective disease surveillance and better define activity space to enable more precise prediction that governs on-target control strategies and intervention measures.

\section{Conclusions}

Increased globalisation has amplified transmission of infectious diseases making it possible to reach almost any part of the world within 24 hours. Hence, early identification of high-risk locations and a better spatial understanding of disease transmission dynamics are critical for targeted intervention strategies. The goal of disease modelling communicable diseases is to offer early and reliable prediction of transmission sites and target locations to devise public health countermeasures that can effectively contain the disease from spreading. Our model consistently yields reliable results for predictions up to 10 days and can therefore aspire to be part of an early warning system.

\section{Acknowledgements}

This paper is the result of research collaboration between Princess Margaret Hospital, Hospital Authority and the Department of Geography at the University of Hong Kong. The research is jointly supported by (i) the Research Fund for the Control of Infectious Diseases Project 10090122 administered by the Food and Health Bureau and the Hong Kong SAR Government, and (ii) the Graduate School of the University of Hong Kong. We are grateful to the following government departments of the Hong Kong Special Administrative Region for data access: Department of Health, Centre for Health Protection, and Hospital Authority. Ethical approval has been obtained from the Kowloon West Cluster Clinical Research Ethics Committee (KW/EX/09-121) and the Institutional Review Board of the University of Hong Kong / Hospital Authority Hong Kong West Cluster (UW 09-414).

\section{References}

Ali M, Emch M, Yunus M, Clemens J, 2009. Modeling spatial heterogeneity of disease risk and evaluation of the impact of vaccination. Vaccine 27, 3724-3729. 
Ali S, Keil R, 2006. Global cities and the spread of infectious disease: the case of severe acute respiratory syndrome (SARS) in Toronto, Canada. Urban Stud 43, 491-509.

Anderson RM, May RM, 1991. Infectious diseases of humans: dynamics and control. Oxford: Oxford University Press.

Armelagos GJ, 1998. The viral superhighway. Sciences 38, $24-$ 30.

Aron JL, 2007. Mathematical modeling: the dynamics of infection. In: Infectious disease epidemiology: theory and practice. Nelson KE, Williams CFM (eds). Sudbury: Jones and Bartlett Publishers, 181-212 pp.

Barrett R, Kuzawa CW, McDade T, Armelagos GJ, 1998. Emerging and re-emerging infectious diseases: the third epidemiologic transition. Annu Rev Anthropol 27, 247-271.

Berry BJL, Griffith DA, Tiefelsdorf MR, 2008. From spatial analysis to geospatial science. Geogr Anal 40, 229-238.

Bowen J, Laroe C, 2005. Airline networks and the international diffusion of severe acute respiratory syndrome (SARS). Geogr J 172, 130-144.

Camitz M, Liljeros F, 2006. The effect of travel restrictions on the spread of a moderately contagious disease. BMC Med 4, 32 .

Census and Statistics Department of the Hong Kong SAR Government, 2001. Introduction to the 2001 population census. Available at: http://www.censtatd. gov.hk/major_projects/2001_ population_census/introduction_to_the_2001_ population_census/index.jsp\#chp1 (accessed on October 2008).

Coen PG, 2007. How mathematical models have helped to improve understanding the epidemiology of infection. Early Hum Dev 83, 141-148.

Connolly MA, Gayer M, Ryan MJ, Spiegel P, Salama P, Heymann DL, 2004. Communicable diseases in complex emergencies: impact and challenges. Lancet 364, 1974-1983.

Donnelly CA, Ghani AC, Leung GM, Hedley AJ, Fraser C, Riley S, Abu-Raddad LJ, Ho LM, Thach TQ, Chau P et al., 2003. Epidemiological determinant of spread of causal agent of severe acute respiratory syndrome in Hong Kong. Lancet 361, 1761-1766.

Dye C, Gay N, 2003. Modeling the SARS epidemic. Science 300, 1884-1885.

Eisenberg JNS, Desai MA, Levy K, Bates SJ, Liang S, Naumoff K, Scott JC, 2007. Environmental determinants of infectious disease: a framework for tracking causal links and guiding public health research. Environ Health Perspect 115, 1216 1223.

Fang LQ, de Vlas SJ, Feng D, Liang S, Xu YF, Zhou JP, Richardus JH, Cao WC, 2009. Geographical spread of SARS in mainland China. Travel Med Infect Dis 14, 14-20.

Held L, Hohle M, Hofmann M, 2005. A statistical framework for the analysis of multivariate infectious disease surveillance data. Stat Model 5, 187-99.
Jefferson T, Foxlee R, Del Mar C, Dooley L, Ferroni E, Hewak B, Prabhala A, Nair S, Rivetti A, 2008. Physical interventions to interrupt or reduce the spread of respiratory viruses: systematic review. BMJ 336, 77-80.

Kwong KH, Lai PC, 2010. Spatial components in disease modeling. In: Computational science and its application (ICCSA 2010). Taniar D, Gervasi O, Murgante B, Pardede E, Apduhan BO (eds). LNCS 6016, 401-412 pp.

Lai PC, So FM, Chan KW, 2009. Spatial epidemiological approaches in disease mapping and analysis. Boca Raton: CRC Press.

Lai PC, Wong CM, Hedley AJ, Lo SV, Leung PY, Kong J, Leung GM, 2004. Understanding the spatial clustering of severe acute respiratory syndrome (SARS) in Hong Kong. Environ Health Perspect 112, 1550-1556.

Lau JTF, Fung KS, Wong TW, Kim JH, Wong E, Chung S, Ho D, Chan LY, Lui SF, Cheng A, 2004a. SARS transmission among hospital workers in Hong Kong. Emerg Infect Dis 10, 280-286.

Lau JTF, Lau M, Kim JH, Wong E, Tsui HY, Tsang T, Wong TW, 2004b. Probable secondary infections in households of SARS patients in Hong Kong. Emerg Infect Dis 10, 235-243.

Lipsitch M, Cohen T, Cooper B, Robins JM, Ma S, James L, Gopalakrishna G, Chew SK, Tan CC, Samore MH et al., 2003. Transmission dynamics and control of severe acute respiratory syndrome. Science 300, 1966-1970.

MacPherson DW, Gushulak BD, Baine WB, Bala S, Gubbins PO, Holtom P, Segarra-Newnham M, 2009. Population mobility, globalisation, and antimicrobial drug resistance. Emerg Infect Dis 15, 1727-1732.

Meade MS, 1997. Medical geography as human ecology: the dimension of population movement. Geogr Rev 67, 379-393.

Meng B, Wang J, Liu J, Wu J, Zhong E, 2005. Understanding the spatial diffusion process of severe acute respiratory syndrome in Beijing. Public Health 119, 1080-1087.

Nelson KE, 2007. Emerging and new infectious diseases. In: Infectious disease epidemiology: theory and practice. Nelson KE, Williams CFM (eds). Sudbury: Jones and Bartlett Publishers, 407-504 pp.

Riley S, Fraser C, Donnelly CA, Ghani AC, Abu-Raddad LJ, Hedley AJ, Leung GM, Ho LM, Lam TH, Thach TQ et al., 2003. Transmission dynamics of the etiological agent of severe acute respiratory syndrome (SARS) in Hong Kong: the impact of public health interventions. Science 300, 1961-1966.

Satcher D, 1995. Emerging infections - getting ahead of the curve. Emerg Infect Dis 1, 1-6.

Shannon GW, Willoughby J, 2004. Severe acute respiratory syndrome (SARS) in Asia: a medical geographic perspective. Eurasian Geogr Econ 45, 359-381.

Sharmin S, Rayhan I, 2011. Modelling of infectious diseases for providing signal of epidemics: a measles case study in Bangladesh. J Health Popul Nutr 29, 567-573. 
Sifat S, Rayhan I, 2012. A stochastic model for early identification of infectious disease epidemics with application to measles cases in Bangladesh. Asia Pac J Public Health, 2012 Nov 18. [Epub ahead of print].

Smith KF, Dobson AP, McKenzie FE, Real LA, Smith DL, Wilson ML, 2005. Ecological theory to enhance infectious disease control and public health policy. Front Ecol Environ 3, 29-37.

Tobler WR, 1970. A computer movie simulating urban growth in the Detroit Region. Econ Geog 46, 234-240.

Trottier H, Philippe P, 2000. Deterministic modeling of infectious diseases: theory and methods. The Internet Journal of Infectious Diseases 1. Available at: http://ispub.com/ IJID/1/2/12783 (accessed on October 2010).

Viboud C, Bjørnstad ON, Smith DL, Simonsen L, Miller MA,
Grenfell BT, 2006a. Synchrony, waves, and spatial hierarchies in the spread of influenza. Science 312, 447.

Viboud C, Miller MA, Grenfell BT, Bjørnstad ON, Simonsen L, 2006b. Air travel and the spread of influenza: important caveats. PLoS Med 3, e503.

WHO, 2003. Severe acute respiratory syndrome (SARS): status of the outbreak and lessons for the immediate future. Available at: http://www.who.int/csr/ media/sars_wha.pdf (accessed on July 2007).

Yang K, Sun LP, Huang YX, Yang GJ, Wu F, Hang DR, Li W, Zhang JF, Liang YS, Zhou XN, 2012. A real-time platform for monitoring schistosomiasis transmission supported by Google Earth and a web-based geographical information system. Geospat Health 6,195-203. 